\title{
Changing perspectives in diabetes: their impact on its classification
}

\author{
T. J. Wilkin
}

Received: 21 January 2007 / Accepted: 5 February 2007 / Published online: 25 April 2007

(C) Springer-Verlag 2007

\begin{abstract}
Type 1 and type 2 diabetes are usually regarded as distinct disorders, but the convergence of their phenotypes over recent years, the relationship of body weight to the risk of type 1 diabetes, the diminishing importance of the type 1 susceptibility genes and the finding of autoantibodies in patients with type 2 diabetes, invite a different interpretation. The possibility that type 1 and type 2 diabetes, rather than being different, are merely poles of a single spectrum, where variation in the tempo of beta cell loss determines age at onset and symptoms at presentation, has important implications. Correct classification is crucial because it directs appropriate treatment and, where available, prevention. This article argues that type 1 diabetes is currently misclassified, provides evidence that insulin resistance drives type 1 diabetes as it does type 2, and proposes how the 'accelerator hypothesis' can be tested in a randomised controlled trial, which could demonstrate, for the first time, the safe and effective prevention of type 1 diabetes.
\end{abstract}

Keywords Aetiology · Accelerator hypothesis . Classification $\cdot$ Clinical trial $\cdot$ Insulin resistance . Type 1 diabetes

\footnotetext{
T. J. Wilkin

Department of Endocrinology and Metabolism, Peninsula Medical School,

Plymouth, UK

T. J. Wilkin $(\bowtie)$

University Medicine, Level 7, Derriford Hospital,

Plymouth PL6 8DH, UK

e-mail: T.Wilkin@pms.ac.uk
}

\author{
Abbreviations \\ LADA latent autoimmune diabetes of adulthood \\ FPIR first-phase insulin release \\ HOMA- homeostasis model assessment for insulin \\ IR resistance \\ NOD non-obese diabetic \\ ENDIT European Nicotinamide Diabetes Intervention \\ Trials
}

\section{Diabetes: two types}

Diabetes used to be straightforward. There was a childhood type that required insulin, and an adult type that did not. Adult-onset diabetes seldom presented before the age of 50 years, and most died with it, rather than of it. Childhood diabetes was readily distinguishable by its acute, ketoacidotic presentation associated with weight loss. Furthermore, insulin levels were high in adult diabetes - a feature of the insulin resistance that was thought to cause it-but low or absent in the childhood disease. The categorisation into two types seemed further justified by three observations, made more than 30 years ago, that applied to childhood, but not to adult diabetes: lymphocytic infiltration of the islets [1], association with immune response (HLA) genes [2], and autoantibodies to islet-related proteins [3]. Childhood diabetes became type 1 or autoimmune diabetes; adult diabetes, type 2 or metabolic.

\section{Diabetes: a continuum}

But cracks soon began to appear in this simple duality. Reports appeared in the late 1970s of adults diagnosed 
with type 2 diabetes who were nevertheless antibodypositive [4]. Mankind is master at reconciling the apparently irreconcilable, and coined the term type $11 / 2$ diabetes to circumvent the difficulty [5]. However, the presence of antibodies in type 2 diabetes predicted future insulin requirement. Some years later, the presence of antibodies in adult diabetes, and the less reactive genotypes associated with it, led to a new disorder of latent autoimmune diabetes of adulthood (LADA) [6]. But the designation was more to do with registers than with reality. Paediatric clinics tend to keep diabetic registers, whereas adult clinics do not, and it took whole-population studies to show that more than half of type 1 diabetes presents over the age of 16 years [7]. The designation of childhood diabetes as a separate entity was, with hindsight, inappropriate, and the basis for LADA arguably unfounded. It nevertheless remains true that those who carry the most intensely reactive HLA genes (those that encode the DR3/4 serotype) tend to present earliest (i.e. in childhood) [8], and that type 1 diabetes later in life is, for the same reason, usually genotypically different, less acute and slower in onset. Type 1 and type 2 diabetes appear to form a continuum, with considerable overlap in age at onset.

This continuum is shifting, and shifting rapidly. Type 2 diabetes, once the preserve of middle age, is now presenting in adolescence $[9,10]$, while type 1 diabetes is increasing most rapidly in children under 5 years of age $[11,12]$. Indeed, diabetes as a whole is now the fastest growing chronic disorder in paediatric practice, and paediatricians are reporting increasing difficulty in distinguishing between the classic phenotypes of type 1 and type 2 [13]. Obese children with acanthosis nigricans (pigmented thickening of the flexures associated with insulin resistance) are presenting with islet-related autoantibodies [14], which were once the diagnostic hallmark of type 1 diabetes. Over the past 30 years, age at onset has moved down the scale to render diabetes an increasingly homogeneous syndrome of obesity, hyperglycaemia, dyslipidaemia and autoantibodies in young people. While the image of the cachectic, ketoacidotic and precomatose child will remain, the reality is changing. A recent report covering an entire population of children presenting with type 1 diabetes between 1980 and 2003 showed a progressive rise in BMI at onset, the age-adjusted mean of which began to exceed that of unaffected children by the mid-1990s [15]. Meanwhile, the number of people with type 2 diabetes requiring insulin is rising.

Insulin independence is not a diagnostic entity, but merely a stage - however brief or extended - through which all diabetic individuals pass on their way to insulin requirement. The insulin-independent stage of type 1 diabetes may be difficult to detect in some children, but is often revealed once the metabolic upset (infection or other acute cause of insulin resistance that temporarily raised beta cell demand beyond its reserve) has settled - the so-called 'honeymoon period' [16]. The stages of diabetes, whether type 1 or type 2 , are the same: prediabetes, insulin independency and insulin dependency.

\section{Implications for the classification of diabetes}

The taxonomy we have adopted for diabetes no longer serves us well, and the failure of a classification to stand the test of time often means that the underlying mechanisms upon which it was based were insecure. A thoughtful and erudite Editorial in this journal recently charted the changes in thinking that have led to present concerns on nomenclature [17]. As the Editor explains, a classification may come to embody outworn concepts that prevent us from seeking or applying new information. Established classification may thus perversely serve to discourage new thinking. The current categories of diabetes include type 1a [18], type $1 \mathrm{~b}$ [19], type $11 / 2$ [5], type 2, LADA [6], MODY 1-6 [20], double diabetes [21], hybrid diabetes and latent autoimmune disease in youth (LADY) [22]. The MODYs are rare single-gene disorders in which the molecular basis for diabetes (impaired glucose sensing or release) has been largely elucidated. Insulin resistance is well established as the driver of type 2 diabetes but, despite 30 years of intensive research based on the autoimmune paradigm, the cause of type 1 diabetes remains unknown, the mechanism of beta cell destruction is not understood, and there is no safe means of preventing it.

Given the difficulty of fitting modern diabetes into its traditional classification, there is arguably reason to review the paradigms on which the classification was initially based. Classification is important to epidemiology and treatment, but classifications that merely grow in complexity as new observations emerge are suspect. Reductionism, which proposes that entities should not be multiplied more than is necessary (Occam's razor), is a rational alternative.

\section{Incidence, age at onset and type of diabetes - the role of tempo}

The increase in incidence and the fall in age at onset of type 2 diabetes over the past generation has been dramatic and widely documented. However, less attention has been paid to the closely similar behaviour of type 1 diabetes over the same interval of time. Type 1 is now two to three times more common than it was a generation ago [12]. Whatever its cause, diabetes is ultimately a disorder of beta cell insufficiency, as loss of glucose control only occurs once the beta cells are unable to supply sufficient insulin for the body's needs [23]. Loss of beta cell function progresses 


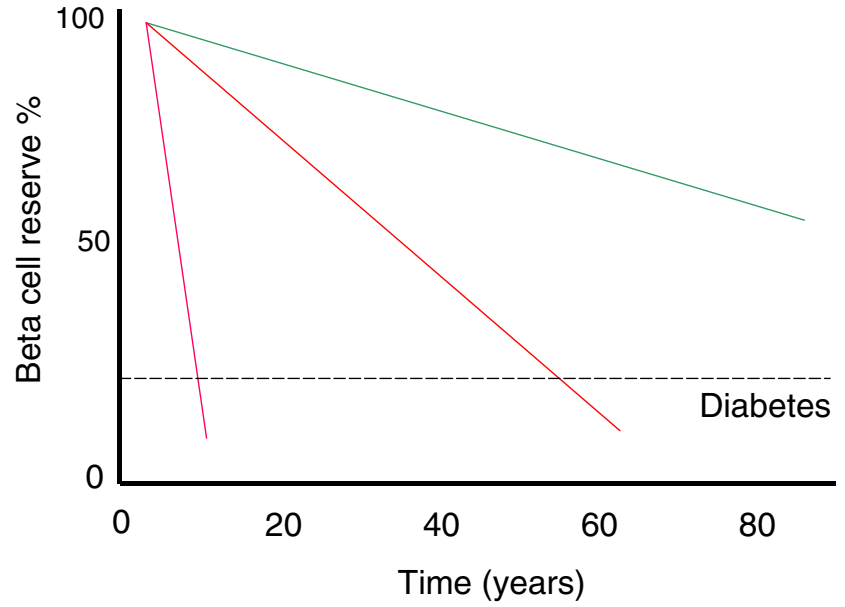

Fig. 1 The concept of tempo in diabetes. Diabetes occurs with a critical loss of beta cell function. Accelerators modulate the tempo of the loss. Most avoid diabetes during their lifetime (green line), but in others the tempo is accelerated sufficiently to develop diabetes in adulthood (orange line) or, in cases of rapid acceleration, childhood (red line). The only difference between the three is one of tempo

naturally over the course of a lifetime as a result of programmed cell death (apoptosis), and blood sugar rises slowly as a result. For most people, the beta cell reserve with which they started life is sufficient to carry them through without diabetes. For others, accelerated loss of beta cells results in diabetes (Fig. 1). The issue for diabetes, whether type 1 or type 2 , is one of tempo and the factors that influence it.

A generation is too short for the gene pool to change, meaning that changing environmental exposure is likely to have been responsible for the quickening tempo of diabetes. A rise in tempo means earlier onset and higher incidence in younger people. Given their parallel behaviour over recent time, it would seem appropriate to ask whether the factor(s) responsible for the rise in tempo might be the same for type 1 and type 2 diabetes.

People with type 1 and type 2 diabetes are often conceived as two separate populations, whereas in reality they both occupy the same obesogenic environment shared by us all. The BMI of the average UK adult has risen from 23 to $26 \mathrm{~kg} / \mathrm{m}^{2}$ over the past generation [24], equivalent to around $9 \mathrm{~kg}$ of mostly extra fat, and there has been an even bigger percentage increase in the BMI of children [25]. One consequence of weight gain is insulin resistance, which demands more and more of the beta cell, and has long since been thought to underlie type 2 diabetes. The metabolic upregulation of beta cells also renders them more antigenic [26], and the acccelerator hypothesis proposes that, in such circumstances, those with reactive HLA genes will mount the immune response that in the past has been interpreted as 'autoimmunity', but is in reality a response to increased antigenicity of the beta cell driven by insulin resistance in genetically susceptible people [27]. If the immune response to metabolic upregulation accelerates beta cell apoptosis to an even greater extent, those with the most immunoresponsive genes may expect to accelerate the fastest and therefore present the earliest. This is the classic picture of childhood diabetes or, more accurately, diabetes in childhood.

HLA-determined susceptibility to type 1 diabetes has been recognised for decades. The finding of a similar association in type 2 diabetes [28], however, supports the hypothesis that HLA susceptibility is not dichotomous but continuous, modulating the tempo of beta cell loss across the spectrum of diabetes. The central concept of the 'accelerator hypothesis' is one in which insulin resistancein its own right an accelerator of beta cell apoptosis-also drives an immune response in consequence, whose intensity is modulated by the HLA genotype. Insulin resistance and HLA genotype are interactive in determining tempo. A highly reactive HLA genotype (e.g. genes that determine the HLA DR3/DR4 serotype) accelerates tempo with little insulin resistance (beta cell upregulation) needed to drive it, while a less reactive genotype needs more. The spectrum linking the two is continuous, and the classic forms of type 1 and type 2 diabetes sit at opposite ends of it.

\section{Key evidence for the accelerator hypothesis}

- The rise in incidence of type 1 diabetes has been contemporaneous with the rise in childhood obesity.

- Children who go on to develop type 1 diabetes are heavier as toddlers than their peers who do not (cause precedes effect - the rule of temporality).

- In a population of children with type 1 diabetes, the fattest present youngest-evidence of true acceleration and, crucially, the only example of 'dose-response' among the many candidate triggers for type 1 diabetes.

- The contribution of high susceptibility genes to the development of type 1 diabetes has diminished over the past generation, consistent with a rising contribution of environmental pressure.

- In meta-analysis, body weight is the only factor that independently influences clinical remission in type 1 diabetes.

- In identical twins at genetic risk of type 1 diabetes, those who go on to develop disease have higher insulin resistance and lower beta cell reserve than those who do not.

- Among children at risk, those with the highest insulin resistance at baseline are those most likely to develop type 1 diabetes subsequently. 


\section{Evidence for the role of insulin resistance in type 1 diabetes}

The suggestion that insulin resistance is the driver behind type 1 as much as type 2 diabetes, and the source of an immune response previously viewed as primary, is challenging but supported by extensive evidence. Not only has the rise in type 1 diabetes paralleled the rise in obesity and that of type 2 diabetes, but children who go on to develop type 1 diabetes are heavier (by implication, more insulin resistant [29]) from an early age than their peers who do not [9, 30-34]. Furthermore, and crucially, five independent studies of children with type 1 diabetes show an inverse relationship between BMI and age at diagnosis [15, 35-38]. Fatter children present earlier-true acceleration.

The probability of developing a multifactorial disease such as type 1 diabetes involves the interaction between genetic susceptibility and environmental risk. Both are proportions. If the proportion of the probability attributable to risk rises, the proportion attributable to susceptibility must fall, and there is good evidence to indicate that the HLA contribution to type 1 diabetes is falling [39, 40]. Correlation with rising obesity over the same period as a fall in heritability makes insulin resistance a prime candidate.

\section{Diabetes: a singular disease}

Perhaps the most striking change in diabetes over recent years has been the convergence of previously distinctive phenotypes. Children who appear clinically to have type 2 diabetes now turn out to have the humoral and cellular responses to islet-related antigens that have traditionally defined type 1 diabetes [14]. Again, convergence is consistent with a hypothesis proposing that type 1 and type 2 diabetes are the same disorder of insulin resistance set against different genetic backgrounds [27]. Progressive loss of the difference in genetic (HLA) susceptibility between type 1 and type 2 diabetes leaves ... the same disorder of insulin resistance.

Witness to the impact of insulin resistance on contemporary type 1 diabetes are the higher doses of insulin now needed to treat people with type 1 diabetes, reports that body weight (insulin resistance) is the only factor to independently influence remission [41], and the demonstration that those at risk who convert to type 1 disease over a period of time (those, by implication, whose tempo is faster) are more insulin resistant from the outset than those who do not [42]. Monozygotic twin pairs from the British
Twin Study were prospectively tested for beta cell reserve (first-phase insulin response [FPIR]) and for insulin resistance (fasting insulin). The co-twins who became diabetic over time had a lower FPIR but higher insulin levels before onset than control subjects, whereas the cotwins who did not become diabetic were no different from control subjects [43].

\section{Limitations and alternative interpretations}

Few of the data presented measure insulin resistance and body fat directly, depending instead on the homeostasis model assessment for insulin resistance (HOMA-IR) and BMI as surrogates. Although most resistance to insulin stems from overweight, BMI is not a measure of insulin action, nor strictly one of fatness. Furthermore, the secular rise in BMI among children over the past generation has arguably occurred alongside other lifestyle and environmental changes that have equal right to be considered among possible risk 'triggers' for type 1 diabetes. Nevertheless, BMI correlates with insulin resistance from early childhood [44], and HOMA-IR is closely related to euglycaemichyperinsulinaemic clamp data in children $(r \geq 0.9)$ [45]. Most importantly, the inverse correlation noted earlier between BMI and age at onset of type 1 diabetes makes body mass unique among the many risk factors proposed for type 1 diabetes. It means that the rising tempo of type 1 diabetes is not merely concurrent with rising body fat, but is linked quantitatively to it in individuals.

It is not within the scope of this review to consider the animal models of type 1 diabetes that are used to support the autoimmunity hypothesis. An immense amount of detailed information on the course of insulitis has been learned from them, and models such as the non-obese diabetic (NOD) mouse and BioBreeding (BB) rat suggest that apparently spontaneous autoimmune reactions can cause diabetes predictably in rodents. They cannot, however, be used to infer that what happens to inbred animals, genetically mutated and confined to a germ-free environment, necessarily explains the process in outbred, freeliving man. Nevertheless, it may be relevant that many of the non-HLA genes differentially regulated in the NOD mouse appear to be more commonly associated with type 2 diabetes than with type 1 diabetes [46].

\section{Refutation}

How could the accelerator hypothesis be tested and, if found wanting, refuted? The hypothesis is based on the 
notion that insulin resistance accelerates beta cell apoptosis both directly and by provoking an immune reaction among those who are genetically susceptible. The logical approach is a randomised controlled trial whose intervention seeks to reduce insulin resistance in children at risk of type 1 diabetes. The experience of the European Nicotinamide Diabetes Intervention Trials (ENDIT) provides an important guide to risk stratification and subject selection for such a trial [47]. The primary outcome variable would be the rate of conversion to diabetes over a specified period, a measure of tempo. Failure to slow the tempo of conversion to type 1 diabetes or other measures of beta cell loss, such as C-peptide or stimulated insulin response, in the face of falling insulin resistance (fasting insulin levels, HOMA-IR), would refute the hypothesis that insulin resistance was responsible for type 1 diabetes. Ability to slow the conversion, accompanied by a fall in antibody titre to suggest diminished intensity of the immune response, would be consistent with the causal relationship proposed here between insulin resistance and the immune reaction to it. Although immunomodulatory therapy (predictably) slows the tempo of beta cell loss [48], insulin re-sensitisation may be an intervention higher upstream that is more physiological, potentially less toxic, and considerably cheaper [49].

\section{Summary points}

- Accurate classification, based on verified mechanisms, is important to guide appropriate treatment and, where available, prevention.

- The clinical phenotypes of type 1 and type 2 diabetes are converging, and the conventional classification of type 1 diabetes as an immunological disorder is increasingly less able to explain its behaviour.

- Insulin resistance leads to the metabolic upregulation of beta cells, which is believed to hasten the tempo of beta cell loss in type 2 diabetes. The accelerator hypothesis proposes that insulin resistance remains the driver responsible for yet faster acceleration of beta cell loss among those with reactive immunoresponse (HLA) genes, and cites evidence to support the mechanism.

- The hypothesis is sufficiently well developed to consider a randomised controlled trial with metformin or thiazolidinedione. Proof of principle that insulin re-sensitisation in people at risk of type 1 diabetes reduces the tempo of beta cell loss would provide a basis for prevention.

- The reclassification of type 1 diabetes into a single disorder alongside type 2 diabetes may encourage a lifestyle approach to the prevention of the most common chronic disorder in childhood.

\section{Conclusion}

Hyperinsulinaemia resulting from insulin resistance appears to precede all forms of diabetes except for the single gene disorders of glucose sensing or insulin release. Nature's weight gain experiment-almost certainly the largest 'clinical trial' of all time-has been associated with an exponential rise in insulin resistance, and in type 1 and type 2 diabetes [50]. Hypotheses need mechanisms to guide the interventions used to test them. The issues here are whether there is now sufficient evidence to justify an intervention study and sufficient benefit from the outcome. The hypothesis that type 1 and type 2 diabetes are the same disorder has a mechanism to explain it, but so far only circumstantial evidence to support it. The perceived outcome-reclassification of diabetes as a single entity - might strengthen resolve to redirect resources towards the prediabetic state, where the opportunity for prevention surely lies. A single disorder, invoking a single approach to prevention, could have considerable impact on the burgeoning problem of childhood diabetes.

\section{References}

1. Gepts W (1965) Pathologic anatomy of the pancreas in juvenile diabetes mellitus. Diabetes 14:619-633

2. Nerup J, Platz P, Anderssen OO (1974) HL-A antigens and diabetes mellitus. Lancet 2:864-866

3. Bottazzo GF, Florin-Christensen A, Doniach D (1974) Islet-cell antibodies in diabetes mellitus with autoimmune polyendocrine deficiencies. Lancet 2:1279-1283

4. Irvine WJ, McCallum CJ, Gray RS, Duncan LJ (1977) Clinical and pathogenic significance of pancreatic-islet-cell antibodies in diabetics treated with oral hypoglycaemic agents. Lancet 1:1025-1027

5. Juneja R, Palmer JP (1999) Type $11 / 2$ diabetes: myth or reality? Autoimmunity 29:65-83

6. Tuomi T, Groop LC, Zimmet PZ, Rowley, MJ, Knowles W, Mackay IR (1993) Antibodies to glutamic acid decarboxylase reveal latent autoimmune diabetes mellitus in adults with a noninsulin-dependent onset of disease. Diabetes 42:359-362

7. Molbak AG, Christau B, Marner B, Borch-Johnsen K, Nerup J (1994) Incidence of insulin-dependent diabetes mellitus in age groups over 30 years in Denmark. Diabet Med 11:650-655

8. Caillat-Zucman S, Garchon HJ, Timsit J et al (1992) Agedependent HLA genetic heterogeneity of type 1 insulin-dependent diabetes mellitus. J Clin Invest 90:2242-2250 
9. Rosenbloom AL, Joe JR, Young RS, Winter WE (1999) Emerging epidemic of type 2 diabetes in youth. Diabetes Care 22:345-354

10. Etisham S, Barrett TG (2004) The emergence of type 2 diabetes in childhood. Ann Clin Biochem 41:10-16

11. Karvonen M, Pitkaniemi J, Tuomilehto J (1999) The onset age of type 1 diabetes in Finnish children has become younger. (The Finnish Childhood Diabetes Registry Group). Diabetes Care 22:1066-1070

12. Gale EAM (2002) The rise of childhood diabetes in the twentieth century. Diabetes 12:3353-3361

13. Fagot-Campagna A, Narayan KMV, Imperatore G (2001) Type 2 diabetes in children. BMJ 322:377-378

14. Brooks-Worrell BM, Greenbaum CJ, Palmer JP, Pihoker C (2004) Autoimmunity to islet proteins in children diagnosed with newonset diabetes. J Clin Endocrinol Metab 89:2222-2227

15. Betts P, Mulligan J, Ward P, Smith B, Wilkin T (2005) Increasing body weight predicts the earlier onset of insulin-dependent diabetes in childhood: testing the 'accelerator hypothesis' (2). Diabet Med 22:144-151

16. Heinze E, Thon A (1985) Honeymoon period in insulin-dependent diabetes mellitus. Pediatrician 12:208-212

17. Gale EA (2006) Declassifying diabetes. Diabetologia 49:1989-1995

18. Babaya N, Nakayama M, Eisenbarth GS (2005) The stages of type 1A diabetes. Ann N Y Acad Sci 1051:194-204

19. Maugendre D, Alizadeh M, Gauthier A et al (1996) Genetic heterogeneity between type $1 \mathrm{a}$ and type $1 \mathrm{~b}$ insulin-dependent diabetes mellitus: HLA class II and TAP gene analysis. Tissue Antigens 48:540-548

20. Owen K, Hattersley AT (2001) Maturity-onset diabetes of the young: from clinical description to molecular genetic characterization. Best Pract Res Clin Endocrinol Metab 15:309-323

21. Libman IM, Becker DJ (2003) Coexistence of type 1 and type 2 diabetes mellitus: "double" diabetes? Pediatr Diabetes 4:110-113

22. Reinehr T, Schober E, Wiegand S, Thon A, Holl R (2006) Betacell autoantibodies in children with type 2 diabetes mellitus: subgroup or misclassification? Arch Dis Child 91:473-477

23. Weir GC, Laybutt DR, Kaneto H, Bonner-Weir S, Sharma A (2001) Beta-cell adaptation and decompensation during the progression of diabetes. Diabetes 50(Suppl 1):8154-8159

24. Prentice AM (1997) Obesity - the inevitable penalty of civilisation? Br Med Bull 53:229-237

25. Bundred P, Kitchiner D, Buchan I (2001) Prevalence of overweight and obese children between 1989 and 1998: population based series of cross sectional studies. BMJ 322:326-328

26. Budde P, Schulte I, Appel A et al (2005) Peptidomics biomarker discovery in mouse models of obesity and type 2 diabetes. Comb Chem High Throughput Screen 8:775-781

27. Wilkin TJ (2001) The accelerator hypothesis: weight gain as the missing link between type I and type II diabetes. Diabetologia 44:914-922

28. Tuomilheto-Wolf E, Tuomilheto J, Hitman GA et al (1993) Genetic susceptibility to non-insulin-dependent diabetes mellitus and glucose intolerance are located in the HLA region. BMJ 307:155-159

29. Voss LD, Kirkby J, Metcalf BS et al (2003) Preventable factors in childhood that lead to insulin resistance, diabetes mellitus and the metabolic syndrome (EarlyBird 1). J Pediatr Endocrinol Metab 16:1211-1224

30. Baum JD, Ounsted M, Smith MA (1975) Weight gain in infancy and subsequent development of diabetes mellitus in childhood. Lancet 2:866

31. Johansson C, Samuelsson U, Ludvigsson J (1994) A high weight gain in early life is associated with an increased risk of type 1 (insulin-dependent) diabetes. Diabetologia 37:91-94

32. Hypponen E, Kenward MG, Virtanen SM et al (1999) Infant feeding, early weight gain and risk of type 1 diabetes. Diabetes Care 22:1961-1965
33. Bruining GJ (2000) Association between infant growth before onset of juvenile type-1 diabetes and autoantibodies to IA-2. (Netherlands Kolibrie study group of childhood diabetes). Lancet 356:655-656

34. Hypponen E, Virtanen SM, Kenward MG, Knip M, Akerblom HK (2000) Obesity, increased linear growth, and risk of type 1 diabetes in children. (Childhood Diabetes in Finland Study Group). Diabetes Care 23:1755-1760

35. Kibirige M, Metcalf B, Renuka R, Wilkin TJ (2003) Testing the accelerator hypothesis: the relationship between body mass and age at diagnosis of type 1 diabetes. Diabetes Care 26:2865-2870

36. Knerr I, Wolf J, Reinehr T, Stachow R et al (2005) The 'accelerator hypothesis': relationship between weight, height, body mass index and age at diagnosis in a large cohort of 9,248 German and Austrian children with type 1 diabetes mellitus. Diabetologia 48:2501-2504

37. Kordonouri O, Hartmann R (2005) Higher body weight is associated with earlier onset of type 1 diabetes in children: confirming the 'accelerator hypothesis'. Diabet Med 22:1783-1784

38. Dabelea D, D'Agostino RB Jr, Mayer-Davis EJ et al (2006) SEARCH for Diabetes in Youth Study Group. Testing the accelerator hypothesis: body size, beta-cell function, and age at onset of type 1 (autoimmune) diabetes. Diabetes Care 29:290-294

39. Hermann R, Knip M, Veijola R (2003) Temporal changes in the frequencies of HLA genotypes in patients with type I diabetesindication of an increased environmental pressure? Diabetologia 46:420-425

40. Gillespie KM, Bain SC, Barnett AH et al (2004) The rising incidence of type 1 diabetes is associated with a reduced contribution from high-risk HLA haplotypes. Lancet 364:1699-1700

41. Scholin A, Torn C, Nystrom L et al (2004) Normal weight promotes remission and low number of islet antibodies prolong the duration of remission in type 1 diabetes. Diabet Med 21:447-455

42. Fourlanos S, Narendran P, Byrnes GB, Colman PG, Harrison LC (2004) Insulin resistance is a risk factor for progression to type 1 diabetes. Diabetologia 47:1661-1667

43. Hawa Ml, Bonfanti R, Valeri C, Delli Castelli M, Beyan H, Leslie $\mathrm{RD}$ (2005) No evidence for genetically determined alteration in insulin secretion or sensitivity predisposing to type 1 diabetes: a study of identical twins. Diabetes Care 28:1415-1418

44. Wilkin TJ, Metcalf B, Jeffery A, Howdle S, Kirkby J, Voss LD (2002) The relative contributions of birth weight, catch-up weight and current weight to the development of insulin resistance in contemporary children (EarlyBird 2). Diabetes 51:3468-3472

45. Gungor N, Saad R, Janosky J, Arslanian S (2004) Validation of surrogate estimates of insulin sensitivity and insulin secretion in children and adolescents. J Pediatr 144:47-55

46. Chaparro RJ, Konigshofer Y, Beilhack GF, Shizuru JA, McDevitt HO, Chien HY (2006) Nonobese diabetic mice express aspects of both type 1 and type 2 diabetes. Proc Natl Acad Sci USA 103:12475-12480

47. Gale EA, Bingley PJ, European Nicotinamide Diabetes Intervention Trial (ENDIT) Group (2006) Progression to type 1 diabetes in islet cell antibody-positive relatives in the European Nicotinamide Diabetes Intervention Trial: the role of additional immune, genetic and metabolic markers of risk. Diabetologia 49:881-890

48. Keymeulen B, Vandemeulebroucke E, Ziegler AG et al (2005) Insulin needs after CD3-antibody therapy in new-onset type 1 diabetes. N Engl J Med 352:2598-2608

49. Wilkin TJ, Ludvigsson J, Greenbaum CJ, Palmer JP, Becker DJ, Bruining J (2004) Future trials for the prevention of type 1 diabetes. Diabetes Care 27:996-997

50. Wilkin TJ (2006) The great weight gain experiment, accelerators and their implications for autoantibodies in diabetes. Arch Dis Child 91:456-458 